\title{
ARTICLES
}

\section{AGAINST HOMEOPATHY - A UTILITARIAN PERSPECTIVE}

\section{KEVIN SMITH}

\section{Keywords \\ homeopathy, alternative medicine, complementary medicine, CAM}

\begin{abstract}
I examine the positive and negative features of homeopathy from an ethical perspective. I consider: (a) several potentially beneficial features of homeopathy, including non-invasiveness, cost-effectiveness, holism, placebo benefits and agent autonomy; and (b) several potentially negative features of homeopathy, including failure to seek effective healthcare, wastage of resources, promulgation of false beliefs and a weakening of commitment to scientific medicine. A utilitarian analysis of the utilities and disutilities leads to the conclusion that homeopathy is ethically unacceptable and ought to be actively rejected by healthcare professionals.
\end{abstract}

What is the moral status of homeopathy? I shall address this question by exploring and weighing the benefits (or utilities) of homeopathy with its disutilities. From this analysis it will be concluded that a strong rejection of homeopathy is morally required, with implications for the ethical behaviour of a range of agents involved in healthcare.

\section{HOMEOPATHY: A PARADOX}

Homeopathy as a claimed therapeutic modality occupies a paradoxical position in modern medicine and healthcare: the plausibility of homeopathy is entirely untenable on logico-scientific grounds, and no quality evidence exists to support claims of efficacy; despite this, homeopathy is manifestly popular amongst many laypeople and a significant number of medical professionals.

Because this paradox exists in the context of medicine and healthcare, with concomitant implications (good or bad) for human welfare, the moral content of homeopathic theory and practice demands analysis. Such an evaluation is of prima facie relevance for a wide range of disparate agents, including public healthcare purchasers, medical practitioners, university educators and private individuals.

\section{BACKGROUND TO HOMEOPATHY}

The basic principles of homeopathy were formulated by a German physician named Samuel Hahnemann in the early 19th century. ${ }^{1}$ Homeopathy is based on two central principles: the 'law of similars' and the 'law of infinitesimals'. The former principle holds that a substance able to cause a symptom in healthy subjects can also be used to cure that symptom. The latter principle holds that a therapeutic substance becomes more potent as it is diluted, provided that the process of dilution is accompanied by a special form of vigorous shaking ('succussion'). Hahnemann and his followers assembled a body of literature based on observations of the apparent effects of administration of a range of diluted substances on various subjects (including themselves). ${ }^{2}$

The fundamental principles and knowledge-base of homeopathy remain essentially unchanged since the 19th century, and form the basis of current homeopathic practice. But these principles are highly problematic, as discussed below.

\footnotetext{
${ }^{1}$ S. Hahnemann \& C. Hering. 1849. Organon of Homoeopathic medicine. New York: W. Radde.

${ }^{2}$ O.W. Holmes ed. 1892. Homeopathy. New York: Prometheus Books: 221-243.
} 


\section{Law of similars}

Hahnemann derived this law from a single observation involving himself. In an attempt to discover why quinine relieves the symptoms of malaria, he took some cinchona bark (the source of quinine) and developed a fever and other symptoms common to malaria. From this experience, he concluded that a substance that produces particular symptoms in healthy individuals can be effective against a disease that has the same symptoms. Subsequently, Hahnemann and his early followers conducted 'provings' in which they administered various substances (of botanical, animal or mineral origin) to themselves and other healthy people, keeping detailed records of the associated symptoms observed. These observations have been compiled into reference works or 'Homeopathic Materia Medica', which form the basis of current homeopathic practice. $^{3}$

To determine whether a given substance actually causes a symptom, it would be necessary to conduct a clinical trial in which people who receive the substance are compared with people who receive a control substance. To minimise the risk of false results through random effects, a fairly large number of people would have to be enrolled on such a trial, since it is commonplace and entirely natural for healthy people to experience occasional unpleasant bodily feelings. Finally, to avoid bias, the trial would have to be 'double-blinded' (i.e. neither the experimenters nor the test subjects should be aware of which people receive the test substance or the control substance).

The 'provings' of Hahnemann et al. were conducted in the early 19th century, an era in which the fundamental principles of clinical trials (as outlined above) had not been established. Thus, the observations that form the basis of current homeopathic practice were not conducted in a reliable fashion. Specifically, participants were observed on an individual (rather than group trial) basis; there were no controls; wide variations occurred in the treatment regimes (in terms of quantities of substance administered, source and preparation of substances, and length of study); blinding was not used; and the 'data' were recorded in a non-rigorous manner. Moreover, many of the symptoms recorded are highly subjective. For example, 'griping pains followed by passing offensive flatus, or a loose yellowish stool, which always produced great exhaustion' (Echinacea angustifolia), 'excited in

\footnotetext{
${ }^{3}$ Hahnemann himself published several volumes of 'Materia Medica Pura' during the early 19th Century. These volumes have been translated and remain in use today. Also available are various Homeopathic Materia Medica works presented on websites as searchable compendiums, for example W. Boericke. 1927. Homoeopathic Materia Medica. 9th edn. Médi-T. Available at: http://www.homeoint.org/books/ boericmm/index.htm [Accessed 21 June 2010]; J.H. Clarke. 1901. A Dictionary of Practical Materia Medica. Médi-T. Available at: http:// www.homeoint.org/clarke/a.htm [Accessed 21 June 2010].
}

evening in bed as if intoxicated, and feeling as if the head were floating in the air' (Juglans regia), and 'singing, tinkling, and buzzing in ears' (Oleum animale). ${ }^{4}$ Further, it is likely, given the lack of blinding, that suggestibility played an important role in the reporting of such effects.

Thus, the process by which the 'materia medica' of homeopathy were established is of extremely poor quality by the standards of modern clinical trials, to the extent that it is impossible to know whether the recorded symptoms were causally related to the administered substances. Thus, no worthwhile knowledge upon which to base medical practice may reliably be taken from these 'provings'.

More fundamentally, there is simply no plausible or rational basis for supposing that substances that produce particular symptoms can cure the same symptoms. The assumption that 'like cures like' places the onus on proponents of homeopathy to elucidate the special logic involved: to the knowledge of the present author, a satisfactory account of this logic has not been made.

\section{Law of infinitesimals}

Homeopathic preparations are produced by taking a quantity of a substance associated with disease symptoms (from the 'provings' information referred to above) and subjecting this to a simple process known in science as 'serial dilution'. In this process, 1 part of the substance is diluted in 9 or more parts of diluent (usually water or alcohol); 1 part of this diluted preparation is then diluted in the same way, with this procedure being repeated several times. ${ }^{5}$ Each stage of dilution is prepared by a special form of shaking ('succussion') that is believed by proponents to increase the potency of the product (the resultant preparation is said to be 'potentized'). ${ }^{6}$ Final dilution factors vary, but are typically in the range of 1 part in $1 \times 10^{6}$ to 1 part in $1 \times 10^{60}$, although some preparations are as high as 1 part in $1 \times 10^{400}$ (that is, 1 part in 1 followed by 400 zeros). ${ }^{7 \dagger}$ The high dilution factors inherent in homeopathic preparations present a fundamental problem, because straightforward mathematics shows that, in most cases, it is statistically unlikely that even a single molecule of the original substance will be

\footnotetext{
† Note: Correction added on 16 August 2012 after first publication online on 14 February 2011. The dilution factor has been corrected from $1 \times 10^{200}$ to $1 \times 10^{400}$ to match the description in the text. The error has been corrected in this version of the article. ${ }^{4}$ Ibid.

5 The precise methods used vary according to the substance in question. Insoluble substances are typically ground-up and mixed (diluted) with milk sugar. Final preparations may be in liquid form or impregnated onto sugar pills.

${ }^{6}$ S. Hahnemann. 1846. The Chronic Diseases their Specific Nature and Homeopathic Treatment. Toronto: Bastian Books.

${ }^{7}$ S. Barrett. 2002. Homeopathy's 'Law of Infinitesimals'. Homeowatch. Available at http://www.homeowatch.org/basic/infinitesimals.html. [Accessed 21 June 2010].
} 
present in the final preparation taken by the patient. For example, to receive just one molecule from a fairly standard homeopathic dilution of $1 \times 10^{30}$, the patient would have to consume over 30,000 litres of the homeopathic solution. ${ }^{8}$

Empirically, pharmacology has demonstrated that physiological responses are dose-dependent. It is implausible to expect the opposite, namely that diluting a substance could increase its effectiveness: reducing the quantity of an active substance to close to zero ought to decrease, not enhance, the activity of that substance; and effectively eliminating the substance (as is frequent in homeopathic dilutions) ought to remove its activity. To suggest otherwise appears to run counter to fundamental logic. Similarly, there is no known or plausible way in which shaking a substance during dilution can increase its potency.

Some modern homeopaths deal with this epistemic problem by employing the ad hoc notion that the water used for dilution retains a 'molecular memory' of the original substance. ${ }^{9}$ However, the idea that water (or any other diluents, such as alcohol) has a 'memory' is unsubstantiated by any known laws or mechanisms of chemistry or physics. More fundamentally, if it were true that water can 'remember' (perhaps by a yet-to-be-discovered revolutionary new scientific mechanism), then every substance ever encountered by a molecule of water - such as food molecules, toxins, heavy metals - should be expected to imprint a 'memory' that would exert powerful and unpredictable effects on the body. The fact that physiological chaos does not ensure whenever we drink a glass of water provides a simple but powerful refutation of the 'memory' concept in homeopathy.

Some proponents of homeopathy have sought to explain the apparently illogical relationship between homeopathic dilution and efficacy by drawing an analogy with immunization: vaccines contain small amounts of active substance that may, in original form, cause disease. Thus, it is suggested that homeopathic effects may be mediated by the immune system. ${ }^{10}$ However, vaccines contain much larger quantities of active substance than do homeopathic preparations. Moreover, the active substances in vaccines are directly quantifiable and elicit a

\footnotetext{
${ }^{8}$ The implications of homeopathic dilutions are derivable from basic arithmetic considerations, and have been expressed by a number of authors; see for example S. Barrett (ibid.); E. Ernst. Is Homeopathy a Clinically Valuable Approach? Trends Pharmacol Sci 2005; 26: 547-548; B. Goldacre 2008. Bad Science. London: Fourth Estate: 33-36.

${ }^{9}$ For example see L.R. Milgrom. Conspicuous by its Absence: the Memory of Water, Macro-entanglement, and the Possibility of Homeopathy. Homeopathy 2007; 96: 209-219; M. Molski. Quasi-quantum Phenomena: the Key to Understanding Homeopathy. Homeopathy 2010; 99: 104-112.

${ }^{10}$ For example see P. Bellavite et al. Immunology and Homeopathy: the Rationale of the 'Simile'. Evid-based Complement Altern Med 2007; 4: 149-163.
}

measurable response (production of antibodies), features that do not apply to homeopathic preparations. Finally, the analogy fails in respect of the altogether disparate usages of vaccines and homeopathic medicines. Immunization is preventive, unlike homeopathy which is used to treat existing ailments.

\section{Homeopathy research}

I have argued above that homeopathy as a therapeutic system not only lacks plausibility, but runs counter to fundamental principles of science and rationality. Accordingly, it would be astonishing if clinical trials of homeopathy demonstrated any efficacy beyond placebo effects, and indeed to date the best clinical evidence has demonstrated no efficacy. ${ }^{11}$ Nevertheless, isolated reports of apparently successful treatments with homeopathy have been published. However, such reports may properly be accounted for by several factors, as follows.

Firstly, even amongst the highest quality clinical trials (of any forms of drug or intervention), occasional 'false positive' results occur, simply through chance statistical effects. ${ }^{12}$ Secondly, many clinical trials of homeopathy are rendered invalid by serious flaws, such as low subject numbers, poor design and slipshod execution. (In contrast with clinical trails of conventional medicines, homeopathic trials tend to be poorly funded and undertaken on a very limited scale.) ${ }^{13}$ Thirdly, papers reporting positive effects of homeopathy tend, for reasons of plausibility and quality, to be rejected by mainstream medical journals, appearing instead in, at best, low-ranking journals or, most frequently, in specialist 'alternative medicine' journals. ${ }^{14}$ Because the raison d'etre of the latter type of journals is inextricably tied to a belief in 'alternatives' such as homeopathy, a self-referential situation occurs which is permissive to the acceptance of weak or flawed reports of homeopathic clinical effectiveness. Additionally, meta-analyses of multiple homeopathy papers are frequently flawed for similar reasons. ${ }^{15}$

${ }^{11}$ E. Ernst. Homeopathy: What Does the 'Best' Evidence Tell Us? Med $J$ Aust 2010; 192: 458-460; W. Sampson. Homeopathy Does Not Work. Altern Ther Health Med 1995; 1: 48-52.

12 J.P.A. Ioannidis. Why Most Published Research Findings are False. PLos Med 2005; 2: 696-701.

${ }^{13}$ Reviewed in W.B. Jonas et al. A Systematic Review of the Quality of Homeopathic Clinical Trials. BMC Complement Altern Med 2001; 1: 12 For an example of a specific case see K.L. Overall \& A.E. Dunham. Homeopathy and the Curse of the Scientific Method. Vet $J$ 2009; 180: 141-148.

${ }^{14}$ T. Caulfield \& S. DeBow. A Systematic Review of how Homeopathy is Represented in Conventional and CAM Peer Reviewed Journals BMC Complement Altern Med 2005; 5: 12; K.R. Smith \& W. Sampson. Word Use and Semantics in Alternative Medicine: a Survey of Editors of Medical and Related Journals. Medscape J Med 2008; 10: 125.

${ }^{15}$ E. Ernst. A Systematic Review of Systematic Reviews of Homeopathy. Br J Clin Pharmacol 2002; 54: 577-582. 
The Cochrane Collaboration ${ }^{16}$ has conducted a number of systematic reviews into various homeopathic treatments. Each review considers all trials published on a given homeopathic treatment. The reviews cover homeopathy for asthma, ADHD, dementia, influenza, induction of labour and adverse effects of cancer treatments. One review reports positive evidence for the effectiveness of homeopathy. However, this review, which examines homeopathy as a treatment for some of the side-effects of radiotherapy and chemotherapy, concludes that the data are 'preliminary' and the trials 'need replicating'. All the other Cochrane reviews on homeopathy conclude that there exists no good evidence in support of the efficacy of homeopathy. ${ }^{17}$

From Hume onwards, it has been accepted that extraordinary claims require extraordinary evidence. Considering the extent to which the fundamental tenets of homeopathy run counter to established rules of science and reason, empirical evidence in support of homeopathy would have to be particularly robust, in view of the fact that acceptance of homeopathy would entail a major epistemic scientific revolution.

\section{Popularity of homeopathy}

Despite the overwhelming quanta of reason and evidence running counter to claims in its favour, homeopathy remains popular amongst many laypeople and a significant number of healthcare professionals. ${ }^{18}$ Along with several other forms of 'Complementary and Alternative Medicine' (CAM), homeopathy has become big business worldwide. Many patients seek private homeopathic consultations or pressure their physicians for referrals. It is not uncommon for family medical practitioners to recommend or provide CAM, including homeopathic remedies. ${ }^{19}$

Although a detailed consideration of the reasons behind the paradoxical prominence of homeopathy and other CAM modalities lies beyond the scope of the

\footnotetext{
${ }^{16}$ The objective of the Cochrane Collaboration is to systematically locate and evaluate all the evidence surrounding the effectiveness of given medical treatments, in order to produce high quality, unbiased reviews. The resultant Cochrane Database of Systematic Reviews is generally seen as one of the best sources of information on treatment efficacy.

17 All six reviews are publically available from the Cochrane Library at http://mrw.interscience.wiley.com/cochrane/cochrane_search_fs.html [Accessed 23 June 2010].

${ }^{18}$ For a review of the popularity of CAM (including homeopathy) see R.B. Bausell. 2007. Snake Oil Science. New York: Oxford University Press: 1-6; E. Ernst. Prevalence of Use of Complementary/Alternative Medicine: a Systematic Review. Bull World Health Organ 2000; 78 : 252-257.

${ }^{19}$ For example see T.A. Winnick. Medical Doctors and Complementary and Alternative Medicine: the Context of Holistic Practice. Health 2006; 10: 149-173.
}

present paper, an explanation is likely to reside in the domains of psychology and sociology. ${ }^{20}$ Several factors appear to promote the popularity of CAM, including the manifest failure of conventional medicine to provide effective treatments for many disorders, a desire for forms of treatment that are less invasive or carry fewer side-effects, and opposition to certain dominant features of conventional healthcare such as the power of large pharmaceutical companies and the allegedly 'reductionist' nature of science-based medicine. Such attitudes may be compounded by various cognitive errors, including an over-reliance on anecdotal evidence, wishful thinking and scientific ignorance..$^{21}$

Amongst the various forms of CAM commonly used by citizens in developed countries, homeopathy appears to be more popular than most other modalities, ${ }^{22}$ including acupuncture, Ayurveda and Reiki. However, homeopathy appears to be less popular than chiropractic or herbal medicine. ${ }^{23}$ There has been scant published research into reasons behind the relative popularity of homeopathy versus other forms of CAM. Given that no form of CAM can be less effective than homeopathy (considering the impossibility of biological effects from homeopathic dilutions), and assuming that all the main forms of CAM provide approximately equal levels of psychological benefit, the explanation must lie with social factors such as availability and fashion. But homeopathy contains a number of notable features that probably make it innately very attractive to consumers (or healthcare providers) choosing between CAM options, including simplicity of administration, absence of adverse effects and relatively low cost.

Worldwide, homeopathy is one of the five foremost CAM-based therapeutic systems, the others being Ayurveda, chiropractic, traditional Chinese medicine

\footnotetext{
${ }^{20}$ This is not to imply that all forms of CAM should be rejected a priori. Rather, any claim for effectiveness ought to be considered on its merits. No claim should be rejected because it fits, or fails to fit, a particular paradigm. Instead, scientific criteria and methodology need to be used to seek justified answers to two questions: 'Is it plausible?' and 'Does it work'? For a discussion, see K.R. Smith. Anomalous Therapies and Public Health: A Utilitarian Bioethical Response. Public Health Nurs 2008; 25: 269-277.

${ }^{21}$ For an overview see W. Sampson ed. 2000. The Braid of 'Alternative Medicine’. New York: Prometheus Books: 21-31.

${ }^{22}$ Surveys on the use of CAM frequently include 'lifestyle' modalities such as massage, prayer, yoga and tai chi; however, although these modalities are popular, their categorization as forms of CAM is questionable, in view of their widespread non-therapeutic use. Accordingly, in comparing the popularity of homeopathy with other forms of CAM, these 'lifestyle' modalities have been excluded from the present consideration of CAM methods.

${ }^{23}$ P.M. Barnes et al. Complementary and Alternative Medicine Use among Adults and Children: United States, 2007. Natl Health Stat Report 2008: 1-23; H.Y. Ni et al. Utilization of Complementary and Alternative Medicine by United States Adults - Results from the 1999 National Health Interview Survey. Medical Care 2002; 40: 353-358.
} 
(TCM) and unani (Arabic medicine).$^{24}$ Homeopathy is popular in Europe, as well as in Asia and in North America. Moreover, homeopathy has been integrated into the healthcare systems of many countries, including India, Mexico, Pakistan and Sri Lanka. ${ }^{25}$ The fact that millions of the world's inhabitants are exposed to homeopathy gives particular importance to a consideration of the ethics of this form of medicine.

\section{ETHICS OF HOMEOPATHY: PREMISE FOR THE FOLLOWING ARGUMENTS}

Proponents of homeopathy, including some physicians and a few scientists, simply reject the foregoing critique of homeopathy. The same critical points have been made repeatedly and more extensively elsewhere and are wellknown, ${ }^{26}$ yet homeopathy retains many supporters. However, the fact that some people continue to believe in and advocate or practise homeopathy does not provide good reason to accept that homeopathy may have a rational basis. In face of the impossibility of convincing all proponents that homeopathy is without foundation, in order to make progress in considering the associated ethical implications I shall assume the premise that homeopathic medicines can have no direct biochemical or physiological effects on the body.

\section{BENEFITS OF HOMEOPATHY}

Homeopathy offers several potential benefits, or utilities. These include non-invasiveness and financial costeffectiveness. Acceptance that homeopathy cannot have any direct physiological effects leaves room for the claim that the holistic nature of homeopathy represents a benefit; related to this is the notion that benefits arise from placebo effects generated by homeopathic practice. Finally, allowing agents to exercise their autonomy (to employ homeopathy) may provide benefits for the individual.

\section{Non-invasiveness and cost-effectiveness}

Because no surgery or hazardous pharmaceuticals are entailed, homeopathy represents the epitome of noninvasiveness. However, it can be argued that treatment

\footnotetext{
${ }^{24}$ World Health Organization. 2001. Legal Status of Traditional Medicine and Complementary/Alternative Medicine: A Worldwide Review.

${ }^{25}$ World Health Organization. 2002. WHO Traditional Medicine Strategy 2002-2005.

${ }^{26}$ Examples include Goldacre op. cit. note 8, pp. 28-62; Sampson, op. cit. note 11, pp. 48-52; D. Taverne. 2005. The March of Unreason. New York: Oxford University Press: 43-46.
}

with an entirely inactive preparation is equivalent to non-treatment. Thus, the benefit of non-invasiveness is negated by the disutility of ineffectiveness. This form of argument also applies to the claimed benefit of financial cost-effectiveness: low cost is nullified by noneffectiveness, since ineffective medical treatment is of zero value. Moreover, because homeopathic practice entails financial cost (in terms of premises, facilities and remuneration), this becomes a disutility. Thus, arguments from non-invasiveness and cost-effectiveness appear prima facie untenable.

However, conventional medical practice is not immune from the charge of employing ineffective therapies. Some ailments are very difficult to treat (or are simply untreatable) using any of the therapies in the armamentarium of modern medicine, and in such cases physicians may attempt to help - or mollify - patients by prescribing ineffective medicine. Examples include antibiotics for viral infections, B vitamins for multiple sclerosis and saline injections for various complaints. Other than simply to get the patient to stop complaining, the motive for prescribing ineffective medicine appears to be an attempt to improve the patient's situation by psychological means. This may be part of a 'holistic' approach, or the aim may be to elicit a placebo response (or both). A number of recent surveys indicate that such practice is not uncommon. ${ }^{27}$

Holism and placebos are discussed in more detail in the following sections. It will be argued that placebos are generally less effective than frequently assumed, and that various ethical objections undermine placebo-based medical approaches. For the moment, however, I shall assume that placebo medicine can be effective, and ignore its ethical problems, such as to address the following question: in cases where ineffective medicine is considered to be in the best interests of the patient, which form of such medicine should be prescribed?

The answer to this question seems clear: the ineffective medicine that is least invasive and least expensive should be used. On these criteria, homeopathy scores highly. Thus, in cases where a patient's best interests are deemed to be served by the provision of ineffective medicine, homeopathy should be favoured over more expensive alternatives. For example, instead of prescribing antibiotics for a viral infection, homeopathic tablets ought to be preferred, assuming the latter to be less expensive than the former. Even if the homeopathic medicine is no cheaper than the antibiotic, the lack of side-effects, coupled with avoidance of antibiotic resistance issues, would make homeopathy the preferred option.

\footnotetext{
${ }^{27}$ M. Fassler et al. Frequency and Circumstances of Placebo use in Clinical Practice - a Systematic Review of Empirical Studies. BMC Med; 8:15.
} 


\section{Holism}

The term 'holistic' refers to viewing the whole person in his or her emotional, familial and societal context, underpinned by sufficient consultation time. On the basis that a holistic approach is generally beneficial for patient care, ${ }^{28}$ homeopathy offers substantial benefits where the time and effort expended simply talking to the patient surpasses the norm for conventional (non-homeopathic) consultations. However, this benefit is not unique to homeopathy, as holism can be (and often is) built into conventional healthcare. And homeopathy itself is not necessarily holistic: for example, there is no holism involved in the (now ubiquitous) provision of homeopathic preparations for sale online and in high-street pharmacies. Thus, because it is not uniquely associated with homeopathy, holism cannot provide special grounds for supporting homeopathy.

\section{Placebo effects}

It is well known that placebo effects are commonplace in medicine, and that such effects can benefit patients. For example, pills containing only inert ingredients have been shown to reduce patients' perception of pain, and it is likely that the neurophysiological responses to the active constituents of a painkilling drug are frequently accompanied by some additional placebo effects. Thus, placebo effects may be of value in healthcare. ${ }^{29}$ By extension, it can be argued that homeopathy has value purely as a placebo. However, this argument is open to two serious classes of objection, one empirical and the other ethical.

The empirical form of objection to homeopathy-asplacebo concerns the nature and value of placebo effects per se. Although it is beyond the scope of the present paper to explore in detail the literature on placebo effects, key features of these effects are discernable, as follows. ${ }^{30}$

Firstly, placebo effects are clearest for subjective, selfreported symptoms, such as those of pain and depression. This is not to say that biological responses are not obtained through placebo effects. For example, the placebo mechanism of action for pain reduction probably involves activation of the brain's endogenous opioid

\footnotetext{
${ }^{28}$ It should however be noted that holism is not without its drawbacks: overly holistic approaches risk medicalizing problems that are better addressed outside the consulting room, with the associated risk of rendering patients excessively dependent upon healthcare practitioners.

${ }^{29}$ For example see D.G. Finniss et al. Biological, Clinical, and Ethical Advances of Placebo Effects. Lancet 2010; 375: 686-695; F.G. Miller et al. Ethical Issues Concerning Research in Complementary and Alternative medicine. J Am Med Assoc 2004; 291: 599-604.

30 A. Harrington. 1999. The Placebo Effect: An Interdisciplinary Exploration. Cambridge, Mass.: Harvard University Press; A. Hrobjartsson \& P.C. Gotzsche. Is the Placebo Powerless? An Analysis of Clinical Trials Comparing Placebo with No Treatment. $N$ Engl J Med 2001; 344: 1594-1602.
}

system. ${ }^{31}$ However, it is implausible to suppose that neuropsychological mechanisms could be effective against profound pathological processes. ${ }^{32}$ Thus, a placebo is unlikely to show significant effects against (for example) tumour progression.

Secondly, apparent placebo benefits are frequently coincidental with the natural course of the disorder (for example, the symptoms of migraine, the common cold or arthritis) - indeed patients naturally tend to present to physicians when their symptoms are at a peak and hence about to subside naturally. ${ }^{33}$ Thus, placebo effects frequently appear strongest with the first use of a therapy; subsequent uses of the same therapy may deliver diminishing positive responses in the patient. Additionally, various psychological factors are likely to contribute to the time-dependent attenuation of effectiveness. Such factors include a desire for improvement, and the positive expectation thereof. The magnitude of response to a placebo will dissipate if the subjective effectiveness of the therapy was less than initially anticipated by the patient.

These empirical features of placebo effects indicate their limited usefulness, a situation confirmed by a number of studies into placebos. ${ }^{34}$ Placebos are most effective for short-term, subjective symptoms, such as episodic pain; they are much less effective (or are ineffective) for conditions with a profound pathological basis, such as infectious diseases or cancer. This reduces although does not fully refute - the power of the homeopathy-as-placebo argument.

Ethical problems present further difficulties for the homeopathy-as-placebo argument. Placebo-only treatments depend axiomatically on the patient being led to believe an untruth: that the proffered treatment actually causes a physiological change. Thus, homeopathy-asplacebo would have to be based upon, in effect, lying to patients. Non-utilitarian systems of morality generally object fundamentally and strongly to lying: those who follow such ethical approaches would therefore reject homeopathy-as-placebo. By contrast, for most utilitarians, lying can in principle be accommodated where it generates positive utility. From this the following argument can be made: hoodwinking patients (by leading them to believe that homeopathic preparations cause physiological changes) may be outweighed by the health

${ }^{31}$ J.G. Craggs et al. The Dynamic Mechanisms of Placebo Induced Analgesia: Evidence of Sustained and Transient Regional Involvement. Pain 2008; 139: 660-669; J.K. Zubieta \& C.S. Stohler. Neurobiological Mechanisms of Placebo Responses. In Year in Cognitive Neuroscience 2009. 198-210.

${ }^{32}$ For instance see J.C. Coyne et al. Psychotherapy and Survival in Cancer: The Conflict between Hope and Evidence. Psychol Bull 2007; 133: 367-394.

${ }^{33}$ For an interesting discussion of factors associated with this phenomenon see Bausell, op. cit. note 18, pp. 37-57.

${ }^{34}$ A. Hrobjartsson \& P.C. Gotzsche. Placebo Interventions for all Clinical Conditions. Cochrane Database of Systematic Reviews. 2010. 
benefits (via placebo effects) accruing from homeopathy. However, patient autonomy is a key feature of utilitarianism: put simply, maximum utility flows from permitting competent patients to consent (or otherwise) to medical interventions. Autonomy requires the positive provision of full information (hence the term informed consent'): therefore, causing patients to believe an untruth (that homeopathic preparations alter physiological functioning) serves as a substantial disutility by restricting patient autonomy. I suggest that the higher order utilitarian concept of autonomy trumps the narrower utility of immediate patient benefit: if this assertion is correct, then the homeopathy-as-placebo argument appears fatally damaged.

However, it is not clear that homeopathic practitioners are lying in the usual sense. Homeopathic literature does not claim that the modus operandi of homeopathy is elicitation of a placebo response, and it may be assumed that most homeopathic practitioners are not setting out deliberately to hoodwink their patients. By analogy, it would seem erroneous for an atheist to claim that a Christian was lying about the existence of God. It can thus be argued that because the charge of lying (in the usual sense) does not apply, homeopathy is not undermined by ethical arguments against deception..$^{35} *$

This argument is, however, problematic. Its flaw lies in the confusion of agents (the homeopaths) and actions (the delivery of effective medicine). Agents can act in good faith but fail to recognize that the basis for their actions is erroneous. An example would be the physician who believes (contrary to the evidence) that HIV is not the causative agent of AIDS, and thus recommends to her HIV-positive patients that they take vitamins instead of antiretroviral drugs. The HIV-denying doctor is not lying in the usual sense, but her patients have nevertheless been led to believe false information. By extension, homeopathy-as-placebo involves the promulgation of erroneous information amongst patients, even when the homeopath is acting in good faith. In making an ethical evaluation here, the negative consequences, in terms of a violation of patient consent, to some extent conflict with the deontological judgement that the homeopath is acting ethically, i.e. in good faith. From a utilitarian perspective, the consequences are paramount, and thus for utilitarians the homeopathy-as-placebo argument is likely to remain unpersuasive regardless of any positive ethical evaluation of the homeopathic practitioner.

Moreover, in the medical context, in contrast to the religious context, simply acting in good faith is not

* Note: Correction added on 14 March 2011 after first publication online on 14 February 2011. The word 'not' was missing from the sentence on two instances. The error has been corrected in this version of the article.

${ }^{35} \mathrm{I}$ am grateful to the anonymous reviewer who suggested this argument. enough. Healthcare professionals - mainstream or homeopathic - have a positive moral duty to ensure that the treatment of patients is based upon sound evidence and theory. This presents a problem for homeopathy, considering its extremely weak and contentious logicoscientific basis. To extend the religious analogy: a religiously motivated physician who insisted in treating his patients only by intercessory prayer (another CAM) ought to be prevented from doing so. To permit the physician to continue this practice would be ethically reprehensible, regardless of the fact that he truly believes in prayer as the best form of therapy. Similarly, homeopaths acting on the basis of their sincere (but unfounded) beliefs are not lying in the usual sense; nevertheless their behaviour remains ethically unacceptable.

For mainstream healthcare professionals who, unlike homeopaths, do not believe that homeopathic preparations can have any direct biological effects, any decision to supply or support homeopathy-as-placebo would appear to amount to lying in the usual sense. It is difficult to allow, from either a utilitarian or deontological perspective, that such deceptive behaviour could be ethically acceptable.

It would be implausible to claim utilitarian grounds for an absolute prohibition on placebo medicine. Special instances may be discerned where the pursuit of a placebo effect is ethically justifiable (or indeed may be ethically required). Two examples, both concerning pain relief, demonstrate this. Firstly, research has established that painkilling medication works best where the patient has a high expectation of the efficacy of the medication. ${ }^{36}$ Thus, it is difficult to argue against the practitioner choosing to display strong confidence in the prescribed medication, as a deliberate attempt to induce a placebo response. A second example is the significant pain relief that can be obtained by injecting a patient with saline, provided that the patient is duped into thinking that the saline is actually an analgesic drug. ${ }^{37}$ This might apply in a battlefield scenario, where wounded soldiers require pain relief but morphine supplies have run out. Again, it is difficult to find arguments against such placebo-based intervention.

However, these examples do not provide strong grounds for ethical approval of homeopathy-as-placebo. In the first example, the patient is not being told a lie: the practitioner is correct in suggesting that the analgesic medication will have a direct effect on the patient's pain. Any additional 'boost' via the placebo effect is simply an added (and welcome) bonus. Accordingly, patient consent and autonomy are not jeopardized in such cases. The second example concerns an unusual emergency situation where no effective medicine is available, and

\footnotetext{
${ }^{36}$ M.P. Jensen \& P. Karoly. Motivation and Expectancy Factors in Symptom Perception - a Laboratory Study of the Placebo-Effect. Psychosom Med 1991; 53: 144-152.

37 For example see A. Pollo et al. Response Expectancies in Placebo Analgesia and their Clinical Relevance. Pain 2001; 93: 77-84.
} 
represents an action of last resort. It is difficult to find arguments that normal medical practice should be based on actions of last resort. However, it can be argued that routine healthcare does in fact have to deal with situations analogous to the battlefield example. This is exemplified by the terminally ill patient for whom the severe side-effects of conventional therapy (such as chemotherapy) outweigh the benefits in terms of quality of life. Perhaps the best interests of such patients would be best served by rejecting chemotherapy in favour of homeopathy-as-placebo? While there is clear logic to adopting this kind of palliative approach, it seems clear that conventional analgesia would be a better alternative to homeopathy. Homeopathy can only provide placebobased relief, whereas conventional analgesia can provide direct (biochemical) pain relief plus placebo-based enhancement of pain relief.

\section{Autonomy}

An interesting situation arises when fully informed, competent patients nevertheless opt for homeopathy. Such patients cannot benefit medically, other than through limited placebo effects; and they may risk their health through failing to access conventional medical treatment, amounting to a disutility. However, permitting such persons the freedom to exercise their autonomy to employ homeopathy yields at least two benefits. Firstly, the foregoing pro-autonomy argument applies here also: if utility is generally maximized by allowing patient autonomy, then patients should be free to choose homeopathy. Secondly, exercise of autonomy provides psychological benefits for the agent: it is well accepted that denial of free choice, or coercion, results in reduced happiness in the subjects concerned. Moreover, allowing people to make mistakes is arguably a valuable part of personal development, thus serving as a utility. Nevertheless, limiting freedom to choose homeopathy remains a conceptual possibility. Access to many pharmaceutical drugs is restricted in most countries, with the aim of preventing patient harm. In utilitarianism, cases of paternalism vs. autonomy are notoriously difficult to decide, given the difficulties of quantifying the utility and disutility involved. Arguably the best approach is to restrict freedom only in cases where there exists a very high risk of serious accidental self-harm. The danger to health from homeopathy is restricted to the risk of failure to seek conventional healthcare; there can be no direct dangers from the homeopathic preparations per se. ${ }^{38}$ Accordingly,

\footnotetext{
${ }^{38}$ While proper homeopathic dilutions will render the preparation inactive and thus unable to cause harm, the possibility remains that in practice the dilution process may not be carried out adequately, or contaminants might be present in the diluent. Such errors could in principle result in toxicity.
}

I conclude that restriction of individual freedom in respect of homeopathy is not warranted on utilitarian grounds.

\section{The benefits of homeopathy: Conclusions}

It seems clear that the benefits of homeopathy are rather minimal. Several arguments in favour of homeopathy turn out to be invalid. These include arguments based on cost-effectiveness, non-invasiveness and holism. Arguments for homeopathy-as-placebo are weakened by the empirical realities of placebo effects, and seriously undermined by problems of autonomy and informed consent. However, restricting personal freedom in respect of homeopathy is not supported by utilitarian reasoning.

\section{DISUTILITIES OF HOMEOPATHY}

Several potential disutilities are associated with homeopathy. One such disutility has already been touched upon in the foregoing discussion on autonomy and paternalism, namely the risk of failing to seek conventional healthcare. Additional potential disutilities include waste of medical resources, inappropriate indications of efficacy from respected sources, and a general weakening of support for science-based medicine.

\section{Risk of failing to seek conventional healthcare}

The most obvious potential disutility associated with homeopathy arises where patients seek homeopathic remedies instead of conventional medicine. Indeed, the WHO recently warned against the use of homeopathic remedies for serious diseases such as tuberculosis or HIV. ${ }^{39}$ Given the impossibility of direct (non-placebo) effects from homeopathic preparations, it is clear that a patient who could benefit from conventional medicine, but instead opts for homeopathy, will suffer harm through omission. As argued above, the argument from autonomy provides grounds for permitting an informed, competent adult to opt for homeopathy despite a risk of damage to the individual's health, at least where the harm is likely to be minor. However, it is arguable that some (competent) patients may not be reliably informed about the ineffective nature of homeopathy. Evidence suggests that some CAM practitioners, including some homeopaths, may act as proponents of their therapeutic doctrine, rather than sources of reliable advice. ${ }^{40}$ It seems clear that such behaviour is ethically unacceptable.

${ }^{39}$ O. Mashta. WHO Warns against Using Homoeopathy to Treat Serious Diseases. Brit Med J 2009; 339.

${ }^{40}$ For example see K. Schmidt \& E. Ernst. Aspects of MMR - Survey Shows that some Homoeopaths and Chiropractors Advise Against MMR. Brit Med J 2002; 325: 597-597. The ethical aspects of such behaviour are considered in E. Ernst. Advice Offered by Practitioners of 
The argument from autonomy in favour of homeopathy cannot support the use of homeopathy for patients who are unable to give informed consent. In this respect it is ethically concerning that homeopathic remedies are frequently used with children and occasionally with noncompetent adults (for example those with dementia). ${ }^{41}$ Where such usage is simply adjunctive to conventional medicine, the ethical problems of harm though omission do not exist. However, any harm arising from failure to employ conventional medicine in children or noncompetent adults would be ethically indefensible.

\section{Waste of resources}

Those charged with allocating public healthcare resources are obliged to select the most cost-effective forms of medicine. Considering that homeopathy represents an intrinsically ineffective form of treatment, any resources expended on homeopathy represent a waste of resources that could otherwise have been expended on more effective healthcare. The utilitarian logic is clear: such expenditure is unethical.

Homeopathic preparations are (or should be) inexpensive, since they contain no active agents. However, homeopathy is associated with additional costs, including the cost of homeopathic consultations and the provision of specialist hospitals and clinics. Moreover, when no clinical benefit is obtained from homeopathy, patients are likely to return to conventional medicine: in this way, the public purse pays twice.

In reality, not all medical problems can be treated successfully. It is arguably better to discuss with such patients why there may be little that can be given to cure or improve their problems, rather than wasting limited resources on ineffective homeopathic treatments.

\section{Unwarranted credence}

Public healthcare providers may face patient demands to fund homeopathy, and private providers have an incentive to meet the same demands from paying clients. Similarly, healthcare practitioners, whether publicly or privately remunerated, may experience patient pressure towards the provision of homeopathy. Beyond the abovementioned ethical reprehensibility of allocating resources

Complementary/Alternative Medicine: An Important Ethical Issue. Eval Health Prof 2009; 32: 335-342.

${ }^{41}$ U. Altunc et al. Homeopathy for Childhood and Adolescence Ailments: Systematic Review of Randomized Clinical Trials. Mayo Clin Proc 2007; 82: 69-75; R. McCarney et al. Homeopathy for Dementia. Cochrane Database Syst Rev 2003: CD003803; E.A. Thompson et al. The Use of Homeopathic Products in Childhood: Data Generated over 8.5 Years from the Avon Longitudinal Study of Parents and Children (ALSPAC). J Altern Complem Med 2010; 16: 69-79. to ineffective treatments, a broader disutility arises wherever acquiescence is afforded to such demands and pressures: that of sending an unwarranted message of official 'approval' for homeopathy. If respected healthcare agencies (such as the NHS in the UK or the NIH in the US) and the professionals within such organizations appear to support homeopathy, it seems inevitable that laypeople will tend to assume, quite wrongly, that homeopathy is a valid medical modality. This is likely to result in a positive-feedback effect, in which increasing provision of homeopathy feeds demand for more of the same.

Such increased demand is also likely to be felt in the domain of education. Indeed several universities in the UK, US and elsewhere have in recent years launched degree-level education and training in CAM (with homeopathy on the syllabus), including degrees majoring in homeopathy. ${ }^{42}$ While some of these courses may claim to offer a 'critical' approach, the reality is that most simply advocate CAM/homeopathy in a non-analytical fashion. ${ }^{43}$ Indeed it is difficult to conceive of substantive educational provision in homeopathy that could be based on anything other than training students to appreciate and apply it in practice. This inherent tendency towards the indoctrination of students in favour of homeopathy (and other CAM approaches) is ethically questionable. On a broader note, the greater the number of university courses teaching homeopathy, the greater will be the perceived respectability of homeopathy as a form of medicine.

In conclusion, inappropriate 'approval' for homeopathy serves as a disutility, as it will tend to magnify several negative outcomes, namely resource wastage, risk of patients failing to seek effective healthcare, and reduced patient autonomy through the promulgation of erroneous medical knowledge.

\section{Weakening of support for science-based medicine}

A wider problem flows from any tacit 'approval' of homeopathy given by respected sources such as healthcare providers, medical practitioners and university educators: insofar as homeopathy is recognized as an 'alternative' form of medicine, the risk is that credence lent towards this and other antiscientific forms of medicine may contribute to a general weakening of support for science-based medicine. The history of medicine is one of evolution from pre-scientific modalities to increasingly

\footnotetext{
42 J.J. Brokaw et al. The Teaching of Complementary and Alternative Medicine in US Medical Schools: A Survey of Course Directors. Acad Med 2002; 77: 876-881; M.S. Wetzel et al. Courses Involving Complementary and Alternative Medicine at US Medical Schools. J Am Med Assoc 1998; 280: 784-787.

${ }^{43}$ D. Colquhoun. Science Degrees without the Science. Nature 2007; 446: 373-374. W. Sampson. The Need for Educational Reform in Teaching about Alternative Therapies. Acad Med 2001; 76: 248-250.
} 
effective and safe forms of medicine, and this has been achieved primarily through the application of scientific understanding and methodology to health and disease. Although it is impossible to quantify the extent to which a weakening of societal commitment to science-based healthcare may lead to a reduction in the rate of medical improvement, it seems clear that any such weakening must count as a disutility.

\section{Weakening of support for genuine 'complementary' therapies}

Some truly 'complementary' methods exist and have value. Such methods include some forms of meditation, relaxation, massage, music and exercise. Techniques of this type can be employed to relax the patient generally, may reduce symptoms, and often provide forms of psychological support. These genuinely complementary approaches have uses in specific contexts. For example, complementary methods may be offered in palliative care settings when cure or clinical response is not sought, the aim simply being to improve the subjective wellbeing of the patient. ${ }^{44}$

Patients or practitioners who are sceptical about homeopathy are likely to consider all 'complementary' therapies in the same negative light. It is thus possible that, proportional to the extent that homeopathy is prevalent within a healthcare system, the more genuine complementary methods may suffer unwarranted neglect. Where complementary approaches are to be funded within a public healthcare system, expenditure on homeopathy represents a diversion of resources away from genuinely useful complementary therapies into an ineffective and ethically fraught modality. ${ }^{45}$

\section{The disutilities of homeopathy: Conclusions}

Investment in homeopathy by public healthcare providers is unethical as it entails a waste of resources. More widely, a set of disutilities arise when respected agencies and agents support homeopathic provision. These disutilities operate by promulgation of false knowledge, potentially leading to unwarranted individual and societal support for antiscientific modalities of medicine, at the expense of genuine progress in healthcare. Although it is difficult or impossible to quantify the extent of the negative consequences stemming from credence being afforded to homeopathy, I suggest that the effect is important and amounts to a serious net disutility.

\footnotetext{
${ }^{44}$ For example see P.J. Mansky \& D.B. Wallerstedt. Complementary Medicine in Palliative Care and Cancer Symptom Management. Cancer $J$ 2006; 12: 425-431.

${ }^{45}$ D.M. Shaw. Homeopathy is where the Harm is: Five Unethical Effects of Funding Unscientific 'Remedies'. J Med Ethics; 36: 130-131.
}

\section{SHOULD CLINICAL TRAILS OF HOMEOPATHY BE CONDUCTED?}

It can be argued that some medically important therapies may remain undiscovered if homeopathy is not researched. Although this claim appears prima facie correct, it needs to be tempered in at least two ways. Firstly, credible scientific assessments of many claims for the efficacy of several homeopathy treatments already exist, and (as discussed earlier) the results have all been negative or highly equivocal. Secondly, investment in research into homeopathy implies that there is likely to be something medically worthwhile to investigate, or to be gained from such research. Thus, the act of formal investigation lends respectability to homeopathy. An analogy with parapsychology may be useful here: some research into claimed phenomena such as extra-sensory perception or clairvoyance has been conducted, however results are rarely clear cut in any one experiment, and random or erroneous results produce a real danger of lending credence to paranormal belief.

A prime criterion for evaluating medical research proposals is logico-scientific validity. Because homeopathy is based on principles that are incompatible with wellestablished science, investigation of homeopathic treatments appears to be unwarranted on this criterion. In response, it may be claimed that by not conducting such research, the possibility of making revolutionary discoveries in science (such as there being a basis for water having a 'molecular memory' of drug molecules) would be closed off. However, this claim is based on a questionable view of the scientific process. Although scientific theories are rarely set in stone, and occasional 'paradigm shifts' have occurred in the history of science, major changes to fundamental scientific principles require substantive empirical evidence, garnered by extensive and painstaking research involving large numbers of scientists. Highly surprising outcomes from individual studies, i.e. results conflicting with core scientific precepts, can be explained in one of two ways: either the relevant fundamental scientific theory is flawed and requires overhauling, or there is a problem with the research itself. It should be self-evident that the latter explanation is substantially more probable than the former.

It follows that research into homeopathic treatments is ethically contentious, at least where human subjects are involved ${ }^{46}$ At best, such research will generate only negative results, which are of minimal interest to medical journals and other disseminators of research findings, and which can be ignored by advocates of homeopathy. Where isolated positive results are obtained in homeopathy research, the best explanation is that a problem exists

\footnotetext{
46 Other than clinical trials, research into homeopathy includes in vitro (cell and tissue culture) studies and trials involving animals.
} 
with the study in question. (For example chance results generated through statistical variation, inadequate controls, a failure to ensure double-blinding, or even fraud.) To appeal instead to a supposed flaw in core scientific theory would necessitate the rejection of (amongst other principles) the particulate theory of matter and the mathematical logic of serial dilution. Nevertheless, such false positive results are open to misinterpretation, and unwarranted credibility thereby afforded to homeopathy. Indeed, the 'research' sections of pro-homeopathic websites, including those of prominent professional homeopathic associations, frequently exhibit a general pattern of citation of isolated positive results. ${ }^{47}$

Allocation of resources represents another ethical issue for homeopathy research. Rigorous trials cost between $\$ 1$ million and $\$ 5$ million each, and between 5 and 20 trials are needed to prove or disprove the effectiveness of each product or method. Expenditure of such amounts on homeopathy would be ethically unacceptable, given the extremely low likelihood of obtaining patient-benefiting results and the effective diversion of funds from more plausible medical research projects.

A final point concerns a feature of published homeopathy research that appears to operate to provide a selfgenerating impetus for yet more homeopathy research. An informal survey of research papers and systematic reviews on homeopathy reveals an interesting pattern present within most of these papers: negative or highly equivocal results are reported, followed by the claim that more research is needed. ${ }^{48}$ In well-founded areas of medical research, 'more research' (funds permitting) into emerging areas is axiomatically a good thing. However, the implausible nature of homeopathy means that such pleas for 'more research' are inappropriate. Considering the disutilities attached to homeopathy research, the ethically correct call might instead be one of 'no more research'.

\section{HOMEOPATHY AS AN ETHICAL PROBLEM}

From the above analysis of homeopathy, it appears indubitable that the quanta of disutilities clearly exceeds the quanta of benefits. Thus, on a utilitarian account, homeopathy ought to be deprecated. The question then becomes: in light of this conclusion, how ought moral agents involved with homeopathy act?

\footnotetext{
${ }^{47}$ Prominent examples include the British Homeopathic Association http://www.britishhomeopathic.org/research/Conditions_where_ positive_evidence.html and the North American Society of Homeopaths http://www.homeopathy.org/research.html [Accessed 21 June 2010].

${ }^{48}$ To the knowledge of the present author, no specific research on this phenomenon has been published; however the reader is invite to peruse (at random) a few homeopathy research papers, to observe that this pattern is almost ubiquitous.
}

Many elements of medicine have no special ethical content, and in such cases the moral decisions open to agents are salient only in respect of how these elements are employed. For example, chemotherapeutic drugs, dialysis machines or hypodermic needles arguably have no inherent ethical features: essentially these are mere tools, and the pertinent moral questions concern only how they ought to be used, upon whom they should be used, whether an alternative treatment might be preferred, and so on. However, a medical tool gains moral content if by its intrinsic nature it is ineffective: for example, a cheap defibrillator that is unable to deliver a sufficient electrical impulse to restart the heart simply cannot be used in a morally acceptable fashion. Such a machine, in the context of medical practice, is ethically unacceptable, and its purchase or use would be morally unjustifiable. In a similar way, homeopathy ought not to be regarded as a mere tool. Because it is inherently ineffective, homeopathy cannot be ethically neutral. It follows that the purchase, deployment or promotion of homeopathy is morally unacceptable. ${ }^{49}$

Homeopathy touches a broad range of agents, including those responsible for public healthcare purchasing, medical practitioners, educationalists, and individual citizens. For those persuaded by the foregoing arguments that homeopathy is ethically unacceptable, a clear moral imperative follows: act such as to avoid utilizing or promoting homeopathy. This obligation embodies both passive and active behaviours, which will vary according to the specific agents involved. For most professionals involved in healthcare, the passive response is likely to be most frequently applicable: healthcare providers ought not to expend resources on homeopathic clinics and treatments; medical and nursing practitioners ought not to refer patients to homeopaths or to practice homeopathy; and educators ought to desist from establishing programmes or course components in homeopathy. However, a more active response will be required at times. Where any of the various agents experience pressure to provide homeopathy, this ought to be actively resisted. Appropriate behaviour in this context entails elucidating the reality of homeopathy - namely its ineffectiveness - in the direction of those who exert pressure for the provision of homeopathy. Thus, health professionals and educators will do well ethically where they endeavour to explain to the public, to patients and to peers why homeopathy ought to be avoided.

\footnotetext{
${ }^{49}$ While there is scope for restoring the morally neutral status of the defibrillator, by repair or redesign, the same cannot be said for homeopathy, since by definition homeopathic preparations must be devoid of biological activity. Repair or redesign of homeopathy is impossible: for example, the inclusion of a medically sufficient concentration of a pharmaceutical agent in a homeopathic remedy would render the preparation non-homeopathic.
} 
The false credence afforded to ineffective medicine through 'approval' being conferred by respected authorities may be a particular problem in the present-day context of mass-access to a rapidly expanding body of medical knowledge. As medical science progresses, the quantity of health-related information is becoming everlarger; and laypeople now have hitherto unparalleled (and increasing) access to much of this information through the Internet. Due to the natural difficulties of gathering reliable knowledge in the face of such informational overload, shortcuts may be taken by those seeking medical information, in the form of assuming that apparently authoritative sources must be correct. Thus, respected sources with an Internet presence, such as universities, hospitals, and medical journals, together with individual scientists and physicians, should be aware that their online material is likely to be particularly influential. The inclusion of material that appears to be supportive of homeopathy on such websites is therefore particularly problematic from an ethical standpoint.

Finally, it is pertinent to ask: do individual citizens have an ethical duty in respect of homeopathy? It was argued earlier in this paper that such agents ought to be free to expend their own private resources on homeopathy. Nevertheless, there exist at least two utilitarian reasons to view such decisions as not being entirely ethically neutral. Firstly, one's personal health is not exclusively a private matter: the patient with an undetected/untreated disease opting for homeopathy in preference to effective healthcare may suffer debility or death, and thus inflicts a significant cost on society. Secondly, by influencing the behaviour of family and friends, private choices can have social consequences. This parallels, on a much smaller scale, the above-mentioned role of respected bodies (such as the NHS) and professionals (such as medical practitioners) in affording false credence to antiscientific medicine when they choose to support or tolerate homeopathy. The magnitude of disutility accruing from these two negative effects may not be very great: it seems unlikely that many seriously ill persons will persist with homeopathy to the exclusion of conventional medicine as their condition worsens; and the influence of one individual's behaviour on others in this context will often be minimal (and certainly does not amount to coercion). Nevertheless, it remains the case that disutility however minimal - flows even from personal decisions to opt for homeopathy, and therefore there exists at least a modest duty on individual citizens to reject homeopathy.

\section{CONCLUDING REMARKS}

I have argued that the moral status of homeopathy is on balance clearly negative. It is pertinent to ask: to what extent may this conclusion apply to other forms of CAM? To provide an answer, it is firstly necessary to recognize that CAM methods exist in a wide array of forms, with disparate underlying principles. (To name but a few CAM methods not previously mentioned in this paper: acupressure, chelation therapy, energy healing, hydrotherapy, iridology, magnetic therapy, naturopathic medicine, osteopathic medicine, reflexology and sclerology.) Thus, each discrete modality would require a separate evaluation to determine its plausibility and (if prima facie plausible) its efficacy. However, what can be stated at present with confidence is that for any CAM system that is as lacking in plausibility as is homeopathy, the foregoing utilitarian analysis must lead to the conclusion that the CAM modality in question ought to be rejected on ethical grounds.

\begin{abstract}
Acknowledgement
The author would like to thank Wallace Sampson, MD (Professor Emeritus, Stanford University, California) and two anonymous referees for valuable comments and criticism.
\end{abstract}

Kevin Smith, PhD, lectures at Abertay University, Dundee. His main interests include medical ethics and the ethics of genetic modification. 